\title{
Desigualdad salarial entre hombres y mujeres en el Gobierno Federal. El caso del Servicio Exterior Mexicano
}

Reyes Sánchez, Carlos

Universidad Veracruzana, México carloreyes@uv.mx

Canal-Martínez, Margarita

Universidad Veracruzana, México mcanal@uv.mx
Resumen - La desigualdad salarial por razones de género constituye una de las características más persistentes en prácticamente todos los mercados laborales a nivel mundial. El presente artículo analiza las diferencias salariales entre hombres y mujeres, tomando como referencia la Secretaría de Relaciones Exteriores. Mediante el uso de pruebas estadísticas que permiten hacer comparaciones entre grupos, se concluye que las desigualdades salariales entre hombres y mujeres se explican a partir de una marcada segregación vertical.

Palabras claves - Equidad de género; distribución salarial; posiciones jerárquicas; Secretaría de relaciones Exteriores; República Mexicana;

Abstract - Gender based wage differentials is widely observed in the world labor markets. This paper studies the wage differences between men and women that works in the Mexican government using as a case of study the Mexican Secretariat of Foreign Affairs. Results from non-parametric statistical hypothe-

\author{
Del-Callejo-Canal, Diana \\ Universidad Veracruzana, México \\ ddelcallejo@uv.mx
}

sis test to compare two related samples suggest that male-female wage differentials are explained because of a high degree of vertical segregation, where men tend to occupy higher ranking and better paying positions than women.

Keywords - gender equality; income distribution; hierarchical positions; Secretariat of Foreign Affairs; Mexico;

\section{INTRODUCCIÓN}

La desigualdad salarial entre hombres y mujeres es un fenómeno mundial que ha sido ampliamente documentado y analizado en distintos países y bajo distintas perspectivas. De igual forma, no son pocas las iniciativas que se han implementado para reducir la disparidad salarial por razones de género, incluyendo su reciente incorporación como parte de los objetivos funda- 
mentales de la Agenda 2030 de las Naciones Unidas para el Desarrollo Sostenible.

El análisis de las desigualdades salariales por género al interior de las dependencias gubernamentales constituye en aspecto relevante, ya que forma parte de las estrategias trasversales establecidas en el Plan Nacional de Desarrollo 2013-2018 (PND), en el cual se señala la urgencia de "fomentar un proceso de cambio profundo que comience al interior de las instituciones de gobierno $(\ldots)$ con el objeto de evitar que en las dependencias de la Administración Pública Federal se reproduzcan los roles y estereotipos de género que inciden en la desigualdad, la exclusión y discriminación" (Gobierno de la República, 2013, p.23).

Por tal motivo, el presente artículo analiza las diferencias salariales entre hombres y mujeres, tomando como referencia la Secretaría de Relaciones Exteriores, que es una de las dieciocho Secretarías de Estado que conforman la Administración Pública Centralizada. Incluye el uso de pruebas estadísticas que permiten hacer comparaciones entre grupos, así como la descomposición por niveles de puesto entre hombres y mujeres.

Es importante destacar que se eligió la Secretaría de Relaciones Exteriores en virtud de que su Programa Sectorial 2013-2018 contempla dentro de sus estrategias: "Desarrollar medidas y acciones a favor de la paridad de género en los cargos públicos. Incorporar acciones afirmativas que faciliten el ascenso de las mujeres en los puestos y rangos de mayor jerarquía, tanto en Cancillería como en el Servicio Exterior Mexicano" (Secretaría de Relaciones Exteriores, 2013, p.38).

\section{DESIGUALDADES SALARIALES POR GÉNERO}

El principio de igualdad de remuneraciones entre hombres y mujeres fue incorporado por primera vez en el Tratado Internacional de VersaIles de 1919, en el que se establece el principio salario igual, por un trabajo de igual valor.

Existen diferentes teorías que explican la desigualdad entre hombres y mujeres. Por ejemplo, Bec- 
Desigualdad salarial entre hombres y mujeres en el Gobierno Federal.

ker (1957) plantea un modelo de discriminación racial en el que los prejuicios respecto a grupos minoritarios de trabajadores con características personales diferentes (como el sexo o la raza) generan diferencias salariales. Por su parte, Phelps (1972) plantea la existencia de información imperfecta y sostiene que los empresarios toman decisiones en función de las características promedio de los distintos grupos (teoría de la discriminación estadística). Por otro lado, Eckel y Grossman (2008) explican la desigualdad entre hombres y mujeres a partir de una mayor aversión al riesgo por parte de las mujeres, lo que provoca que accedan a puestos de trabajo con menores remuneraciones. De manera similar, Niederle y Vesterlund (2007) explican las diferencias salariales como consecuencia de que las mujeres tienden a rechazar ambientes altamente competitivos. Bowles et al. (2005), sugiere que las diferencias salariales tienen su origen en el hecho de que las mujeres presentan una menor capacidad de negociación salarial para sí mismas.
De manera más general, Anker (1997) concluye que las teorías que explican las desigualdades salariales entre hombres y mujeres se pueden agrupar en tres corrientes genéricas: las teorías neoclásicas de capital humano, las teorías de la segmentación de los mercados de trabajo y las teorías feministas ${ }^{1}$.

En cualquier caso, las desigualdades salariales entre hombres y mujeres constituyen una de las características más persistentes en prácticamente todos los mercados laborales a nivel mundial. Éstas también pueden ser analizadas desde la perspectiva de la segregación laboral por género, vertical y horizontal.

La segregación horizontal se refiere a la concentración de hombres y mujeres en diferentes sectores o actividades identificadas como propias de un género o de otro. En estos casos, "la concentración de mano de obra femenina se justifica convencionalmente: existen trabajos que se definen como masculinos $y$ otros femeninos por las

1 Para más información, consultar Roldán-García et al (2012). 
tareas propias de uno u otro género, considerándose que hay trabajos remunerados femeninos, cuyo ejercicio es adecuado para las mujeres, mientras que otros son impropios de ellas" (Gómez, 2001, p. 123).

De conformidad con la Organización Internacional del Trabajo (OIT) "en los países desarrollados, las mujeres se concentran sobre todo en los sectores menos remunerados como la salud y el trabajo social, la educación y otros servicios. Igualmente, en muchos países en desarrollo $y$, en particular, en los de bajos ingresos, las mujeres están sobrerrepresentadas en las actividades agrícolas con alto coeficiente de mano de obra que requieren muchas horas de trabajo, y cuya remuneración suele ser baja o incluso inexistente" (OIT, 2016, p.38).

Por su parte, la segregación vertical hace referencia a una desigual distribución de hombres y mujeres dentro de la estructura jerárquica de un mismo sector ocupacional. Este concepto se encuentra estrechamente vinculado con lo que Morrison et al (1987) define como "techo de cristal" el cual consiste en una barrera "tan sutil que se torna transparente, pero que resulta un fuerte impedimento para que las mujeres puedan moverse dentro de las jerarquías corporativas" (Morrison et al., 1987, p.13). En general, la literatura de referencia coincide en señalar que las mujeres se encuentran poco representadas en los niveles de mayor poder de decisión, retribución salarial, prestigio, etc. De conformidad con la OIC, "un muestreo realizado en México, Argentina, Brasil y Chile indica que un 32 por ciento de las empresas de estos países no tiene a ninguna mujer en puestos directivos; el 28 por ciento tiene una en cargos de alta dirección; el 20 por ciento, a dos; y otro 20 por ciento, a tres o más" (OIT, 2016, p.21).

\section{Metodología}

Los microdatos empleados en el análisis empírico que se presenta en este escrito provienen del Sistema de Portales de Transparencia (SIPOT) de la Plataforma Nacional de Transparencia, e incluyen género, remuneración mensual neta y nombre del puesto de los 3,865 servidores públicos que al 31 de marzo de 2018 se encontraban laborando en la Secretaría de Rela- 
ciones Exteriores ${ }^{2}$. Es importante destacar que esta información es de carácter pública en términos de lo establecido por el artículo 70 de la Ley General de Transparencia y Acceso a la Información Pública.

Se comparó el comportamiento salarial entre hombres y mujeres adscritos a la Secretaría de Relaciones Exteriores del Gobierno Federal utilizando un contraste basado en la diferencia medianas de diferencia de rangos de Wilcoxon al $95 \%$ de confianza. La hipótesis nula $(\mathrm{HO})$ está dada por la afirmación correspondiente a la igualdad entre la mediana salarial de hombres y mujeres, mientras que la hipótesis alternativa $(\mathrm{H} 1)$ sugiere la desigualdad entre ambos niveles salariales ${ }^{3}$.

$H O: \mu d M=\mu d H$

vs

$$
H 1: \mu d M \neq \mu d H
$$

Donde:

$\mu d M=$ Mediana del salario de las mujeres y

$\mu d H=$ Mediana del salario de los hombres.
También se utilizó una prueba de ji-cuadrada al $95 \%$ de confianza para probar si la ocupación por niveles de puesto depende del hecho de ser mujer u hombre.

\section{Resultados}

De conformidad con la base de datos obtenida a través del SIPOT, la Secretaría de Relaciones Exteriores cuenta con 3,865 trabajadores, de los cuales 1,943 son mujeres $(50.3 \%)$ y 1,922 hombres $(49.7 \%)$. La Tabla 1 resume los principales estadísticos descriptivos de tendencia central y dispersión de las remuneraciones netas de todos los servidores públicos del gobierno federal.

Los estadísticos descriptivos más comunes son la media y la desviación estándar. Sin embargo, estos índices únicamente pueden considerarse robustos cuando las observaciones tienen una distribución normal, o cuando menos simétrica. En caso contrario, lo más recomendable es utilizar la mediana y los cuartiles.

2 Para más información, consultar https://consultapublicamx.inai.org.mx/vut-web/.

3 Para más información, consultar Daniel, W. (1990). 
Tabla 1. Operacionalización de variables.

\begin{tabular}{|c|c|c|}
\hline Medidas de Tendencia Central & Mujeres & Hombres \\
\hline Media aritmética & $\$ 21,722$ & $\$ 29,774$ \\
\hline Mediana & $\$ 14,022$ & $\$ 17,234$ \\
\hline $\begin{array}{c}\text { Medidas de Dispersión } \\
\text { Desviación estándar }\end{array}$ & $\$ 18,569$ & \\
\hline Rango & $\$ 8,420-\$ 137,530$ & $\$ 8,420-\$ 145,111$ \\
\hline Rango inter-cuartílico & $\$ 10,598-\$ 24,504$ & $\$ 10,988-\$ 39,433$
\end{tabular}

Fuente: Elaboración propia con datos del SIPOT, utilizando R-Project, versión 3.5.1.

La Tabla 1 permite observar diferencias significativas entre la media y la mediana, por lo que la distribución de los salarios no se comporta de manera simétrica. Los valores extremos hacen que la media aritmética se eleve demasiado y, en consecuencia, los valores de $\$ 29,774$ (hombres) y $\$ 21,722$ (mujeres) no reflejan adecuadamente el "salario promedio" de los trabajadores. En este contexto, la mediana es el estadístico que mejor describe los valores centrales en la distribución de los sueldos, donde se observa que el $50 \%$ de los hombres y mujeres que trabajan en la Secretaría de Relaciones Exteriores, perciben un salario neto igual o menor a $\$ 17,234$ y $\$ 14,022$, respectivamente.
En la figura 1, muestra el histograma de la distribución de los salarios y permite observar que existe una mayor concentración de trabajadores en la parte izquierda de la gráfica (entre los $\$ 10,000$ y $\$ 20,000$ aproximadamente), pero que existen casos, aunque pocos, que se puede extender hasta los $\$ 145,111$.

Figura 1. Distribución de los salarios en la administración pública federal.

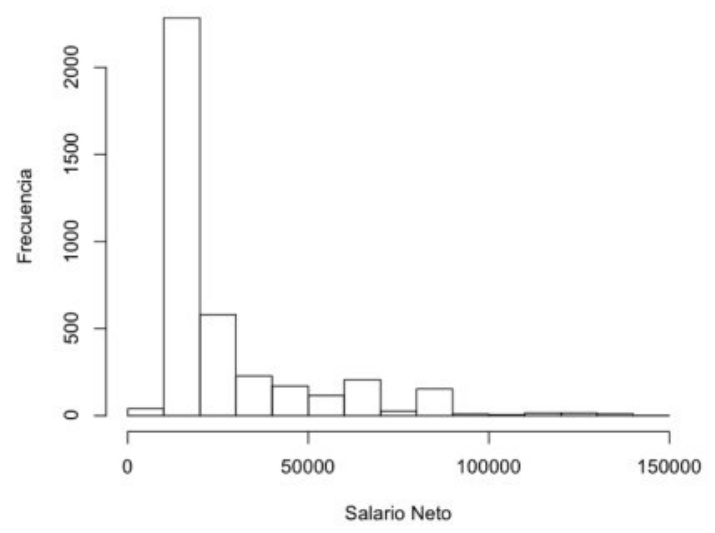

Fuente: Elaboración propia con datos del SIPOT, utilizando R-Project, versión 3.5.1. 
La figura 2 permite comparar las diferencias en la distribución de sueldos entre hombres y mujeres. Se trata de una gráfica de cajas y alambres, en donde se observa que los sueldos tienden a ser mayores en los hombres, que en las mujeres (caja más alta), mientras que el $50 \%$ de los hombres ganan entre $\$ 10,988$ y $\$ 39,433$, el salario del $50 \%$ de las mujeres oscila entre los $\$ 10,598$ y $\$ 24,504$. Los hombres presentan mayor variabilidad (caja más ancha) y, en ambos casos, el "alambre" largo hacia arriba y la línea que divide la caja más hacia abajo (mediana) indican el sesgo a la derecha en la distribución de los salarios, esto es que existe una mayor concentración de trabajadores en los salarios bajos (menores a los $\$ 50,000)$. Los círculos que sobrepasan a los alambres indican la existencia de puntos atípicos. En este caso, esos puntos atípicos corresponden a los niveles más altos dentro de la estructura orgánica de la Secretaría (Secretario de Despacho, Subsecretarios y Directores Generales).

La figura 2 permite ilustrar diferencias salariales de género al interior de la Secretaría de Relaciones

\section{Figura 2. Sueldos en la Secretaría de Relaciones Exteriores, divididos por género.}

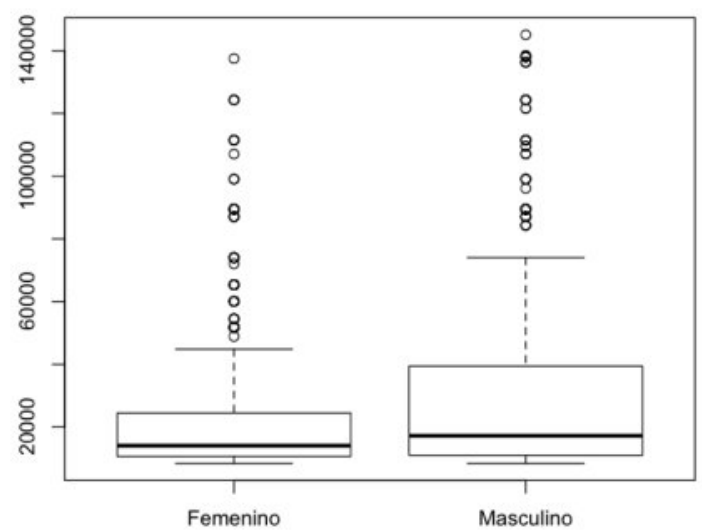

Fuente: Elaboración propia con datos del SIPOT, utilizando R-Project, versión 3.5.1.

Exteriores. A través de la prueba de contraste de Wilcoxon se confirma que efectivamente hay evidencia estadística suficiente para declarar una diferencia entre los salarios de los hombres y los salarios de las mujeres ( $W=155500$, p-valor=0.0001). Un análisis más completo respecto a las diferencias salariales entre hombres y mujeres, se obtuvo a partir de la incorporación de información relativa a la estructura organizacional de la dependencia.

La Tabla 2 permite comparar el número de hombres y mujeres que ocupan cada uno de los niveles jerárquicos dentro de la Secretaría de Relaciones Exteriores. Se obser- 
Tabla 2. Ocupación por niveles de puesto.

\begin{tabular}{l|c|c|c|} 
& Hombres & Mujeres & Total \\
\hline Secretario de Estado & 1 & 0 & 1 \\
\hline Subsecretario & 4 & 0 & 4 \\
\hline Director General & 258 & 93 & 351 \\
\hline Oficial Mayor & 0 & 1 & 1 \\
\hline Jefe de Unidad & 6 & 0 & 6 \\
\hline Director de Área & 173 & 139 & 312 \\
\hline Subdirector & 438 & 368 & 806 \\
\hline Jefe de Departamento & 227 & 311 & 538 \\
\hline Enlace & 286 & 232 & 518 \\
\hline Operativo & 529 & 799 & 1,328 \\
\hline \multicolumn{1}{|c|}{ Total } & 1,922 & 1,943 & 3,865 \\
\hline
\end{tabular}

Fuente: Elaboración propia con información obtenida a través del SIPOT.

va una marcada diferencia en los niveles de Secretario, Subsecretario y Jefe de Unidad, en donde no existe una sola mujer que ocupe dichos cargos. De igual forma, las mujeres únicamente ocupan el $26 \%$ de las plazas de Director General. La prueba ji-cuadrada al $95 \%$ de confianza confirma que la ocupación de niveles por puesto depende del género $\left(x^{\wedge} 2=4997.4, p\right.$-valor $\left.=0.0001\right)$.

Por otro lado, no obstante la segregación vertical que se observa en la Tabla 2, existe evidencia de que si se cumple con el principio de salario igual, por un trabajo de igual valor. Con base en el estadístico de Wilcoxon al $95 \%$ de confian$z a$, se confirma que no existen diferencias significativas entre los sueldos de hombres y mujeres al interior de cada uno de los niveles jerárquicos (ver Tabla 3 ).

En este contexto, el tabulador de sueldos y salarios de la administración del gobierno federal funciona como mecanismo igualador de sueldos entre hombres y mujeres pertenecientes a un mismo nivel jerárquico. Destaca el caso de los 
Desigualdad salarial entre hombres y mujeres en el Gobierno Federal.

Tabla 3. Contraste de diferencia de medianas en Salario, por nivel jerárquico.

\begin{tabular}{|c|c|c|c|}
\hline Nivel Jerárquico & Wilcoxon & p-valor & Interpretación \\
\hline Director General & 12637 & 0.4177 & $\begin{array}{l}\text { No se rechaza Ho, por lo que a } \\
\text { nivel de Director General, no } \\
\text { existen diferencias } \\
\text { significativas entre los sueldos } \\
\text { de hombres y mujeres. }\end{array}$ \\
\hline Director de Área & 11656 & 0.6292 & $\begin{array}{l}\text { No se rechaza Ho, por lo que a } \\
\text { nivel de Director de Área, no } \\
\text { existen diferencias } \\
\text { significativas entre los sueldos } \\
\text { de hombres y mujeres. }\end{array}$ \\
\hline Subdirector & 81208 & 0.8466 & $\begin{array}{l}\text { No se rechaza Ho, por lo que a } \\
\text { nivel de Subdirector, no existen } \\
\text { diferencias significativas entre } \\
\text { los sueldos de hombres y } \\
\text { mujeres. }\end{array}$ \\
\hline $\begin{array}{l}\text { Jefe de } \\
\text { Departamento }\end{array}$ & 36112 & 0.6316 & $\begin{array}{l}\text { No se rechaza Ho, por lo que a } \\
\text { nivel de Jefe de Departamento, } \\
\text { no existen diferencias } \\
\text { significativas entre los sueldos } \\
\text { de hombres y mujeres. }\end{array}$ \\
\hline Enlace & 32540 & 0.6979 & $\begin{array}{l}\text { No se rechaza Ho, por lo que a } \\
\text { nivel de Enlace, no existen } \\
\text { diferencias significativas entre } \\
\text { los sueldos de hombres y } \\
\text { mujeres. }\end{array}$ \\
\hline Operativo & 224030 & 0.05577 & $\begin{array}{l}\text { No se rechaza Ho, por lo que } \\
\text { en puestos operativos, no } \\
\text { existen diferencias } \\
\text { significativas entre los sueldos } \\
\text { de hombres y mujeres. }\end{array}$ \\
\hline
\end{tabular}

Fuente: Elaboración propia con datos del SIPOT, utilizando R-Project, versión 3.5.1. No incluye los niveles de Secretario de Estado, Subsecretario, Oficial Mayor y Jefe de Unidad, toda vez que son todos hombres o mujeres.

puestos operativos, que se encuentra mayoritariamente representado por mujeres $(60 \%$ del total) y cuyo correspondiente $p$-valor se encuentra cercano al umbral establecido en la regla de decisión para el re- chazo o no rechazo de la hipótesis nula. Esto significa que, al interior de este nivel jerárquico, existe un mayor riesgo de equivocarnos, aún y cuando éste se ubica dentro del nivel de confianza del $95 \%$. 


\section{Conclusiones}

Con información transversal al 31 de marzo de 2018 y un nivel de confianza de $95 \%$, se puede concluir la existencia de diferencias salariales significativas entre hombres y mujeres adscritos a la Secretaría de Relaciones Exteriores.

La segregación vertical constituye el principal obstáculo para reducir las diferencias salariales entre hombres y mujeres al interior de la Secretaría de Relaciones Exteriores. Las mujeres tienen una nula representación en los puestos de mayor jerarquía (secretario, subsecretario y jefatura de unidad) y ocupan únicamente el $26 \%$ de los puestos de dirección general. En contraste, las mujeres representan el $58 \%$ de las jefaturas de departamento y el $60 \%$ de los puestos operativos.

En este contexto, se puede concluir que las acciones implementadas por la dependencia no permitieron cumplir con la estrategia establecida en su Programa Sectorial 2013-2018, relativa a la incorporación de medidas y acciones que faciliten ascenso de las mujeres a los puestos de mayor jerarquía, tanto en Cancillería como en el Servicio Exterior Mexicano.

Por otro lado, en cuanto al principio de salario igual, por un trabajo de igual valor, los datos del SIPOT permiten concluir que, para un mismo nivel jerárquico, no existen diferencias significativas entre los sueldos de hombres y mujeres. Es previsible que, al menos una parte, se deba al acatamiento del tabulador de sueldos y salarios.

Finalmente, futuras líneas de investigación sugieren la incorporación de métodos empíricos para estimar mediante análisis de regresión el componente de las diferencias salariales entre hombres $y$ mujeres que no puede ser explicado a partir de las diferencias en los puestos de trabajo o de productividad, así como la incorporación de datos relativos a niveles de escolaridad y experiencia, para analizar si las diferencias salariales efectivamente se asocian a cuestiones de género. 


\section{REFERENCIAS}

Anker, R. (1997). Theories of occupational segregation by sex: An overview. International Labour Review, (136)3, pp. 315-339.

Becker, G. (1957). The Economics of Discrimination. Chicago University Press, Chicago.

Bowles, H., Babcock, L. y Mginn, K. (2005). Constraints and triggers: situational mechanics of gender in negotiation. Journal of Personality and Social Psychology, Vol. 89, pp. 951-965.

Daniel, W. (1990). Applied Nonparametric Statistics. 2nd ed. Boston: PWS-Kent Publishing Company.

Eckel, C. y Grossman, P. (2008). Differences in the economic decisions of men and women: experimental evidence. Plott, Charles, Smith, Vernon (Eds.), Handbook of Experimental Economics Results, Vol. 1, Elsevier, New York.

Gobierno de la República (2013). Plan Nacional de Desarrollo 2013-2018. México. Recuperado de http://pnd.gob.mx/

Gómez, C. (2001). Mujer y trabajo: principales ejes de análisis. Papers 63/64, 123-124. Recuperado de http://dx.doi.org/10.5565/rev/papers/v63n0.1210

Morrison, A.; White, R. \& Van Velson, E. (1987). Breaking the Glass Ceiling: can women reach the top of america's largest corporations? New York, AdisonWesley

Niederle, M. y Vesterlund, L. (2007). Do women shy away from competition? Do men compete too much? Quarterly Journal of Economics Vol. 122, pp.

1067-1101.
OIT Organización Internacioal del Trabajo (2016). Las mujeres en el trabajo: Tendencias de 2016. Recuperado de https://www.ilo.org/wcmsp5/groups/public/---dgreports/---dcomm/-- publ/documents/publication/wcms 483214.pdf

OIT Organización Internacioal del Trabajo (2014). Igualdad de género y no discriminación en la gestión del empleo.

Recuperado de https://www.ilo.org/wcmsp5/groups/public/---americas/---ro-lima/---sro-san iose/documents/publication/wcms 326274 .pdf

ONU Organización de las Naciones Unidas (2018). Agenda 2030 y los Objetivos de Desarrollo Sostenible. Recuperado de https://repositorio.cepal.org/bitstream/handle/11362/40155/10/S1700334 es.pdf

Phelps, E. (1972): The Statistical Theory of Racism and Sexism, American Economic Review, pp. 659-661.

Roldán-García, E., Leyra-Fatou, B. y Contreras-Martínez, L. (2012). Segregación laboral y techo de cristal en trabajo social: análisis del caso español. Portularia, XII(2), pp.43-56. Recuperado de http://www.redalyc.org/articulo.oa?id=161024690004

Secretaría de Relaciones Exteriores (2017). Programa Sectorial de Relaciones Exteriores 2013-2018. Recuperado de https://sre.gob.mx/images/stories/marconormativodoc/dof131213-p.pdf 